\title{
PRIMARY OSTEOSARCOMA OF 7TH RIB IN A 5 MONTH OLD MALE CHILD - AN UNUSUAL PRESENTATION
}

Ramani M, G. Sunitha, Sarika, O. H. Radhika Krishna, K. Ramesh Reddy
1. Professor. Department of Pathology, Niloufer Hospital for Women and Children
2. Post Graduate. Department of Pathology, Niloufer Hospital for Women and Children
3. Post Graduate. Department of Pathology, Niloufer Hospital for Women and Children
4. Assistant Professor. Department of Pathology, Niloufer Hospital for Women and Children
5. Professor \& HOD. Department of Paediatrics Surgery, Niloufer Hospital for Women and Children

\section{CORRESPONDING AUTHOR:}

Dr. Ramani M,

Professor, Department of Pathology,

Osmania Medical College,

Koti, Hyderabad,

Andhra Pradesh, India.

E-mail: drmramani@sify.com

ABSTRACT: Primary osteosarcomas are one of the most common malignant bone tumours in children and adolescents. Most commonly affected bones are long bones and involvement of flat bone in a neonate is quite unusual. We present a case of primary osteosarcoma of $7^{\text {thrib }}$ in a 5 month old male child.

KEYWORDS: Flat bones, Infant, Osteosarcoma, Rib

INTRODUCTION: Osteosarcomas are the most common primary malignant bone tumour exclusive of multiple myeloma and lymphoma. Usually arise in the metaphysis of long bones. ${ }^{1}$ In pediatric age groups common malignant tumour involving chest wall are Ewing's sarcoma, rhabdomyosarcoma and chondrosarcoma. ${ }^{2}$ Primary osteosarcoma in an infant involving the rib is a rare presentation. We present one case of primary osteosarcoma of the 7 th rib in a 5 month old male child.

CASE REPORT: 5 month old male child with history of two berry sized masses in the lower end of chest at the time of birth presented to the outpatient. Mass apparently increased to the present size. Clinical examination revealed 2 distinct bony hard lesions in the lower end of left side of chest. Smaller medial mass measuring $2.5 \times 1.2 \mathrm{~cm}$, lateral mass about $5 \mathrm{~cm}$ from the lower end of sternum measuring about $6.5 \times 5 \mathrm{~cm}$ [Figure 1a]. Clinically diagnosed as Ewing's sarcoma or metastatic deposits of Neuroblastoma.

Routine biochemical parameters were within the normal limits, except serum alkaline phosphate, which was raised upto $963 \mathrm{U} / \mathrm{L}$. Chest X-Ray showed a large soft tissue density in the lower chest wall with splaying of corresponding ribs and a diagnosis of Round cell lesion was made. Ultrasonography of chest was suggestive of multicentric round cell lesion. CT scan chest done showed multiple expansile, mixed sclerotic and lytic lesions of posterior rib cage causing mediastinal invasion and abutting heart with bone destruction [Figure 1b]. No evidence of metastasis. Digital CT performed, showed evidence of expansile lytic lesion arising from the posterior left $7^{\text {th }}$ rib, splaying of $7^{\text {th }}$ and $8^{\text {th }}$ ribs - suggestive of malignant bone lesion of $7^{\text {th }}$ rib Ewing's/neuroblastoma [Figure 2a].In bone scan there was presence of left lower chest osseous lesion. The lesion was accessible for FNAC, so FNAC was performed. FNA cytology smear 
showed plenty of osteoclastic giant cells in the background of osteoid and clusters and singly scattered pleomorphic cells showing hyperchromatic nucleus with prominent nucleolisuggestive of malignant giant cell lesion - Osteosarcoma [Figure 2b].

Biopsy was done for this case and sent for histopathological examination. Grossly received grey brown to grey tan soft tissue bit measuring $1 \times 0.5 \times 0.5 \mathrm{~cm}$. Multiple sections studied from the processed tissue showed fine lace like pink osteoid with areas of trabeculae formation admixed with oval to spindle shaped pleomorphic cells having hyperchromatic nuclei. There were clusters of osteoblastic cells and plenty of osteoclast like multinucleated giant cells suggestive of osteosarcoma [Figure 3]. IHC was performed to confirm the diagnosis. It was positive for vimentin and negative for CD99. CD99 negativity ruled out Ewing's sarcoma. Finally the case was diagnosed as primary Osteosarcoma of rib. The patient has been started on chemotherapy with cisplatin and doxorubicin. With this treatment the tumour mass has regressed in its size.

DISCUSSION: Osteosarcoma is a malignant mesenchymal tumour. It is the most common malignant tumour of bone and accounts for $20 \%$ of bone tumours with a bimodal age distribution. The first peak is in less than 20 years and the second peak is seen in elderly persons. Osteosarcoma usually originates in the metaphysis of the long bones. The usual sites which are affected by tumour are distal femur, proximal tibia and humerus. Long bones have high bone growth and highest mitotic activity because of this unique property, long bones are usually involved. ${ }^{2}$ More than $50 \%$ occurs in the knee region.Most common in males.

Most of the osteosarcoma cases occur denovo, but are also associated with Paget's disease, exposure to radiation, chemotherapy, preexisting benign bone diseases and trauma. Various genetic mutations are involved in the development of osteosarcoma. These are mutation in RB gene. Over expression of p53 and MDM2 is implicated in the genesis of nonhereditary osteosarcoma. ${ }^{3}$

Ewing's sarcoma usually affects chest wall in pediatric age group but Osteosarcoma affecting chest wall bones like a rib is a rare entity. ${ }^{4,5} \mathrm{Few}$ cases have been reported in the literature about primary osteosarcoma of rib in older children of 7 to 13 years of age. 4, 5, 6, 7 Botchu et.al, reported osteosarcoma rib in a 7 year old girl, this was the youngest presentation that was reported in literature. ${ }^{6}$ In our case, the patient is a 5 month old male.

The commonest presentation of osteosarcoma of a rib is pain and palpable mass. In our case, the patient presented with palpable mass. The lesion is usually detected on chest radiography, but typical sun burst pattern that usually observed in osteosarcoma of long bones may not be evident in the flat bones. ${ }^{7}$

CT scan and MRI can evaluate the exact location, extent of tumour and invasion into the deeper structures. In case of posterior rib osteosarcoma, involvement of spinal muscle is best detected by MRI.4 Histopathological examination of mass gives a definite diagnosis. If the tumour produces scant osteoid, it proves difficult for the pathologist. There are various histopathological types of osteosarcoma, these are parosteal, periosteal. Well differentiated intramedullary, osteoblastic, fibroblastic and fibro-sarcomatous type. ${ }^{8}$ The differential diagnosis of osteosarcoma can be ruled out by specific immune histochemical stains. A variety of serological markers have been associated with pediatric osteosarcoma (POS) ${ }^{9}$ [Table 1].

There are various modalities to treat primary osteosarcoma of chest wall, which includes a wide surgical excision followed by neo adjuvant chemotherapy. Surgery should include resection of full thickness of the chest wall with wide margins that may include the 
adjacent ribs, intercostal muscles, pleura and vertebrae. This procedure results in large chest wall defects. So it requires tissue flaps. Titanium mesh is used for this purpose. ${ }^{10}$ Postoperative multi-agent chemotherapy improves the patient survival, reducing the risk of both local and distant relapse. Because of the difficulty of complete excision, the overall prognosis of osteosarcoma in flat bones remains poor. Osteosarcoma of flat bones is rarely associated with distinct metastasis in contrast to osteosarcoma of other sites. ${ }^{4}$

CONCLUSION: To conclude, osteosarcoma of the rib in infants is rare. We present a case of primary osteosarcoma of 7 th rib in a 5 month old male infant. We emphasize that osteosarcoma should be considered in the differential diagnosis of primary malignant neoplasms of chest wall in pediatric age groups. Metastasis at presentation is uncommon, early diagnosis and aggressive surgical management will help to improve the patient's outcome.

\section{REFERENCES:}

1. Mirra JM, Gold RH, Picci P. Osseous tumours of intramedullary origin: In: Mirra JM, editor. Bone Tumours: clinical, Radiological, and Pathological Correlations. Philadelphia: Lea and Febiger; 1989: 143-438.

2. Rosenberg AE. Bones, Joints, and Soft tissue Tumours. In: Robbins, Cotran, editors. Pathological Basis of Disease 7th ed. Philadelphia: Saunders; 2004. . 1273-3243.

3. Leonardo F, et al: p53 and MDM2 alterations in osteosarcoma: correlation with clinicopathologic features and proliferation rates. Cancer. 1997 Apr 15;79(8):1541-7

4. Deitch J, Crawford AH, Choudhury S. Osteogenic sarcoma of the rib: a case presentation and literature review. Spine 2003; 28(4):E74-7.

5. Chattopadhyay A, Nagendhar Y, Kumar V. Osteosarcoma of the rib. Indian J Pediatr 2004; 71(.):543-4.

6. Botchu R, Ravikumar KJ, Sudhakar G et al. Osteosarcoma of rib in a seven-year old child: a case report. Eur J OrthopSurg Traumatology 2006; 16: 156-7.

7. Yamaguchi T, Shimizu K, Koguchi Y et al. Low grade central osteosarcoma of the rib. Skeletal Radiol 2005; 34(8):490-3.

8. Murphey MD, Robbin MR, McRae GA et al. The many faces of osteosarcoma. Radiographics 1997; 17(5): 1205-31.

9. Yulia A Savitskaya, et al:Serum tumor markers in pediatric osteosarcoma: a summary review. Clinical Sarcoma Research 2012, 2:9,

10. WY Lim, et al: Osteosarcoma of the rib. Biomedical Imaging and Interventional Journal: 2008: 4(1):e7.

Table 1: Serological Markers Associated with Pediatric Osteosarcoma

\begin{tabular}{|l|l|}
\hline Serum Marker & Assessed clinical utility of POS \\
\hline VEGF & Prognostic factor \\
\hline KI67 & Prognostic factor \\
\hline TNF $\beta$ \& sTNFR & Monitoring of response to neoadjuvant chemotherapy \\
\hline ANG* & Diagnostic \& Prognostic factor in primary POS \\
\hline Free polyamines & Indicator of malignant process \\
\hline IGF1 \& IGFBP-3 & Predictive factor of development of clinical characteristics \\
\hline SAA $^{* *}$ & Differentiates malignant bone cancer from benign lesion \\
\hline
\end{tabular}


* Angiogenin ** Serum amyloid protein A

Figure 1: (a) 5 month old neonate with left chest wall mass (b)CT showing expansile lesion of posterior rib cage

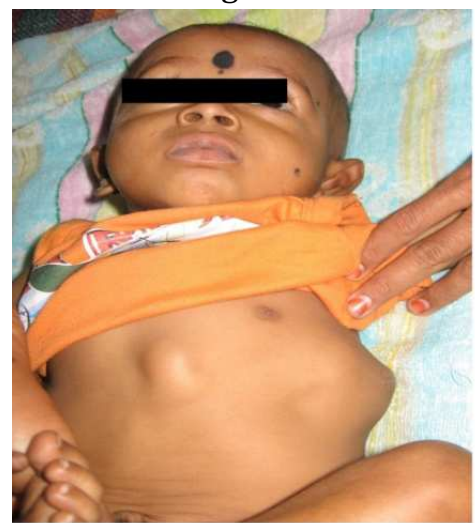

(a)

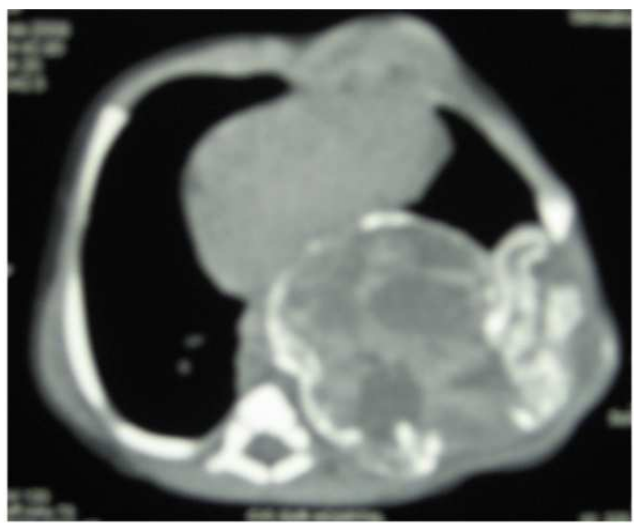

(b)

Figure 2: (a) Digital CT showing expansile lytic lesion arising from $7^{\text {th }}$ rib (b) FNA cytosmear showing pleomorphic tumour tissue cells
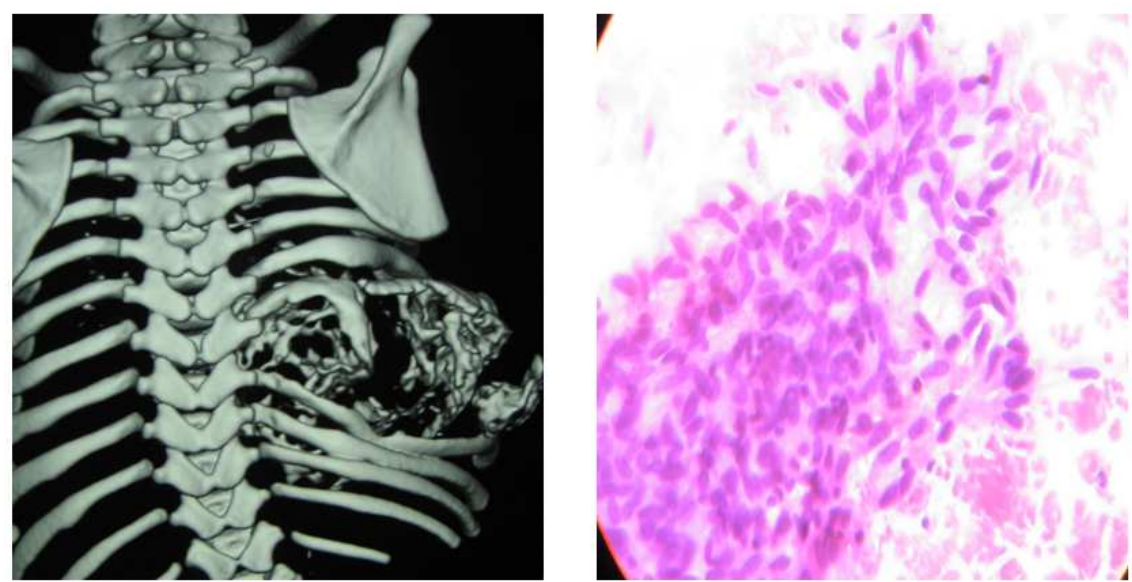

(a) (b)

Figure 3: H\&E section shows lace like osteoid, osteoclast giant cells and tumor cells.
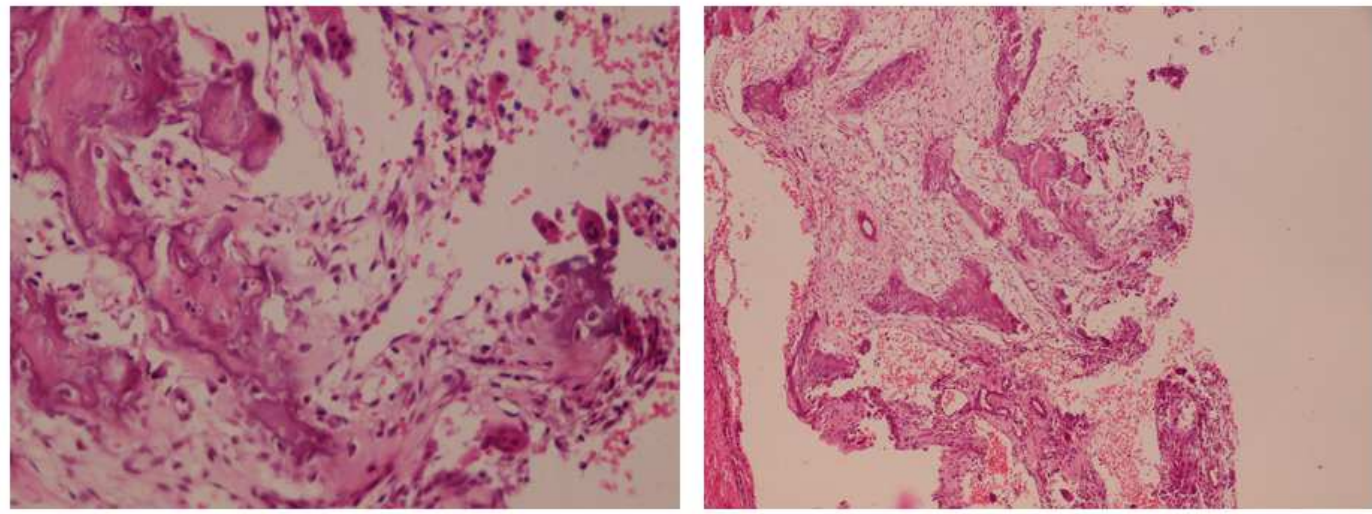